\title{
Stress Potentiates Early and Attenuates Late Stages of Visual Processing
}

\author{
Alexander J. Shackman, ${ }^{2,3 \star}$ Jeffrey S. Maxwell, ${ }^{7 \star}$ Brenton W. McMenamin, ${ }^{8,9}$ Lawrence L. Greischar, ${ }^{1,2}$ \\ and Richard J. Davidson ${ }^{1,2,3,4,5,6}$ \\ ${ }^{1}$ Laboratory for Affective Neuroscience and ${ }^{2}$ Department of Psychology, University of Wisconsin-Madison, Madison, Wisconsin 53706, ${ }^{3}$ Department of \\ Psychiatry, Wisconsin Psychiatric Institute and Clinics, and ${ }^{4}$ HealthEmotions Research Institute, University of Wisconsin-Madison, Madison, Wisconsin \\ 53719, ${ }^{5}$ Waisman Laboratory for Brain Imaging and Behavior and ${ }^{6}$ Center for Investigating Healthy Minds, University of Wisconsin-Madison, Madison, \\ Wisconsin 53705, ${ }^{7}$ Human Research \& Engineering Directorate, United States Army Research Laboratory, Aberdeen Proving Ground, Maryland 21005, and \\ ${ }^{8}$ Department of Psychology and ${ }^{9}$ Center for Cognitive Sciences, University of Minnesota-Twin Cities, Minneapolis, Minnesota 55455
}

Stress can fundamentally alter neural responses to incoming information. Recent research suggests that stress and anxiety shift the balance of attention away from a task-directed mode, governed by prefrontal cortex, to a sensory-vigilance mode, governed by the amygdala and other threat-sensitive regions. A key untested prediction of this framework is that stress exerts dissociable effects on different stages of information processing. This study exploited the temporal resolution afforded by event-related potentials to disentangle the impact of stress on vigilance, indexed by early perceptual activity, from its impact on task-directed cognition, indexed by later postperceptual activity in humans. Results indicated that threat of shock amplified stress, measured using retrospective ratings and concurrent facial electromyography. Stress also double-dissociated early sensory-specific processing from later task-directed processing of emotionally neutral stimuli: stress amplified N1 $(184-236 \mathrm{~ms})$ and attenuated P3 $(316-488 \mathrm{~ms})$ activity. This demonstrates that stress can have strikingly different consequences at different processing stages. Consistent with recent suggestions, stress amplified earlier extrastriate activity in a manner consistent with vigilance for threat (N1), but disrupted later activity associated with the evaluation of task-relevant information (P3). These results provide a novel basis for understanding how stress can modulate information processing in everyday life and stress-sensitive disorders.

\section{Introduction}

Stress is a universal experience that can fundamentally alter neural responses to incoming information. Recent research suggests that stress and anxiety shift the balance of attention away from a task-directed mode, governed by prefrontal cortex (PFC), to a sensory-vigilance mode, governed by the amygdala and other threat-sensitive regions (Bishop, 2007; Arnsten, 2009). A key untested prediction of this framework is that stress exerts dissociable effects across different stages of information processing: potentiating early sensory processing and attenuating later task-directed processing.

Some evidence suggests that threat-elicited stress and anxiety increase vigilance, a sustained enhancement of early sensory pro-

\footnotetext{
Received June 30, 2010; revised Nov. 3, 2010; accepted Nov. 22, 2010.

This work was supported by the Department of Defense (National Defense Science and Engineering Graduate Fellowship to J.S.M.; Army Research Laboratory Director's Strategic Initiative to J.S.M.), National Institutes of Health (F31-MH070110 to J.S.M.; P50-MH52354 and R37-MH43454 to R.J.D.; and support of B.W.M. and A.J.S. through T32-HD007151 and T32-MH18931), and National Science Foundation (Graduate Research Fellowship to A.J.S.). We thank K. Berling, S. Blume, D. Cole, I. Dolski, L. Friedman, J. Koger, J. Nichols, and A. Teche for assistance; and A. Fox, A. Heller, J. Oler, J. E. Shackman, H. Slagter, and two anonymous reviewers for critical feedback.

${ }^{*}$ A.J.S. and J.S.M. contributed equally to this work, and their order was determined by a coin flip.

The authors declare no competing financial interests.

Correspondence should be addressed to Alexander J. Shackman, Jeffrey S. Maxwell, or Richard J. Davidson, University of Wisconsin-Madison, 1202 West Johnson Street, Madison, WI 53706, E-mail: shackman@wisc.edu, jeffsmaxwell@gmail.com, or rjdavids@wisc.edu.

DOI:10.1523/JNEUROSCI.3384-10.2011

Copyright $\odot 2011$ the authors $\quad 0270-6474 / 11 / 311156-06 \$ 15.00 / 0$
}

cessing that facilitates threat detection. Aversive conditioning renders indistinguishable stimuli discriminable ( $\mathrm{Li}$ et al., 2008), threatening stimuli improve contrast sensitivity (Phelps et al., 2006), uncertain physical threats increase risk assessment (Kavaliers and Choleris, 2001), and fearful facial expressions enhance sensory intake (Susskind et al., 2008). These changes may arise from sensitization of the amygdala, which could bias processing directly, via sensory projections (e.g., extrastriate cortex), and indirectly, by modulating ascending neurotransmitter systems (e.g., acetylcholine and norepinephrine) (Davis and Whalen, 2001; Arnsten, 2009; Lim et al., 2009; Sabatinelli et al., 2009).

Other evidence suggests that stress disrupts mechanisms involved in the task-directed regulation of attention and evaluation of task-relevant information, in some cases hampering the performance of tasks that heavily rely on controlled attention, such as spatial working memory (Arnsten and Goldman-Rakic, 1998; Shackman et al., 2006). Performance degradation probably stems from the modulation of task-evoked activity in prefrontal regions (Dolcos and McCarthy, 2006; Erk et al., 2007; Fales et al., 2008; Qin et al., 2009). Such modulation has been ascribed to the diversion of attention (Bishop, 2007) and to changes in the neuronal signal-tonoise ratio that are triggered by the amygdala and mediated by catecholaminergic influences on PFC (Arnsten, 2009).

However, none of these studies has directly assessed the possibility that stress differentially modulates early sensory and later task-directed (postperceptual) stages of information processing, 
as current models hypothesize (Bishop, 2007; Arnsten, 2009). Here, we exploited the temporal resolution of event-related potentials (ERPs) to measure the impact of stress, elicited by taskirrelevant threat of shock, on neural activity evoked by an emotionally neutral visual discrimination task. Stress-induced vigilance were evaluated using the visual P1 (112-144 ms) and N1 (184-236 ms), early sensory-specific ERP components that are generated in extrastriate cortex and sensitive to the amount of attention allocated to incoming information, that is, the signalto-noise ratio of visual processing (Mishra and Hillyard, 2009). Task-directed processing was evaluated using the P3 (316-488 $\mathrm{ms})$, a later component that is largely insensitive to sensory modality and thought to reflect evaluative processes necessary for making task-relevant responses (Nieuwenhuis et al., 2005; Duncan et al., 2009). Stress was verified using facial electromyography (EMG) recorded from the corrugator muscle (supplemental Fig. S1, available at www.jneurosci.org as supplemental material), a well validated measure of negative affect that is sensitive to amygdala activity (Lanteaume et al., 2007; Lee et al., 2009).

\section{Materials and Methods}

Thirty-two (half female) right-handed individuals were recruited and paid $\$ 10 / \mathrm{h}$. Participants provided informed consent in accord with the local Institutional Review Board. At session's start, electrodes were affixed to the fourth and fifth fingers of each hand. Participants were instructed that (1) they would receive one or more painful, but not harmful, electric shocks during threat blocks; (2) the timing and laterality of shocks would be random and unrelated to the task or performance; and (3) they would never receive shocks during safety blocks. Before each block, participants were informed whether it was associated with safety or threat. Shocks (5 mA) were generated using an A13-22 stimulator (Coulbourn Instruments). Participants received 5-6 shocks total. Shock electrodes were unplugged during safety blocks (Grillon and Ameli, 1998).

Task stimuli consisted of up and down target arrows (100 ms; $2.4^{\circ}$ visual angle) presented $5.7^{\circ}$ lateral to fixation (1600-2400 ms stimulus onset asynchrony). Targets were flanked by equiprobable arrow or diamond distractors. Participants were instructed to maintain fixation, respond to targets using corresponding keyboard arrows, and emphasize accuracy over speed. Participants performed sixteen 64-trial blocks (half threat; order counterbalanced). Stimulus presentation was controlled using E-Prime (Psychology Software Tools). Because preliminary analyses indicated that the impact of stress did not vary with distractor type (see the supplemental material, available at www.jneurosci.org), data were collapsed across this factor.

Following the experiment, participants used $100 \mathrm{~mm}$ visual analog scales to rate the intensity of their emotional experience (anxious, happy, safe, or stressed) during safety and threat blocks. To minimize the number of comparisons, a composite stress index was computed by averaging items (positive items reverse-scored; $\alpha_{\text {Cronbach }}=0.78$ ).

EMG and ERP data were acquired using a 128-channel array (Electrical Geodesics; 1-200 Hz bandpass; $500 \mathrm{~Hz}$ digitization; $\mathrm{Cz}$ reference). Data acquisition and preprocessing used Netstation (Electrical Geodesics). EMG was processed following published procedures (Shackman et al., 2006). Segments containing artifacts were rejected. Channels overlying the left and right corrugator muscles (supplemental Fig. S1, available at www.jneurosci.org as supplemental material) were bipolar rereferenced and mean power density $\left(\mu \mathrm{V}^{2} / \mathrm{Hz}\right)$ estimated for the $45-200$ $\mathrm{Hz}$ band using 50\%-overlapped, Hann-windowed 1.024 s epochs. Power densities were $\log _{10}$-transformed and averaged across hemispheres.

ERP data were filtered $(0.3-30 \mathrm{~Hz})$, interpolated to replace unusable channels, and segmented. Segments corresponding to shock delivery, incorrect responses, or artifacts were discarded. Artifact-free data were average re-referenced, baseline corrected $(200 \mathrm{~ms})$, and averaged. Additional processing used EEGLAB (http://sccn.ucsd.edu/eeglab) and inhouse Matlab (The MathWorks) and IDL (ITT Visual Information Solutions) code. Omnibus changes in neural activity were quantified by

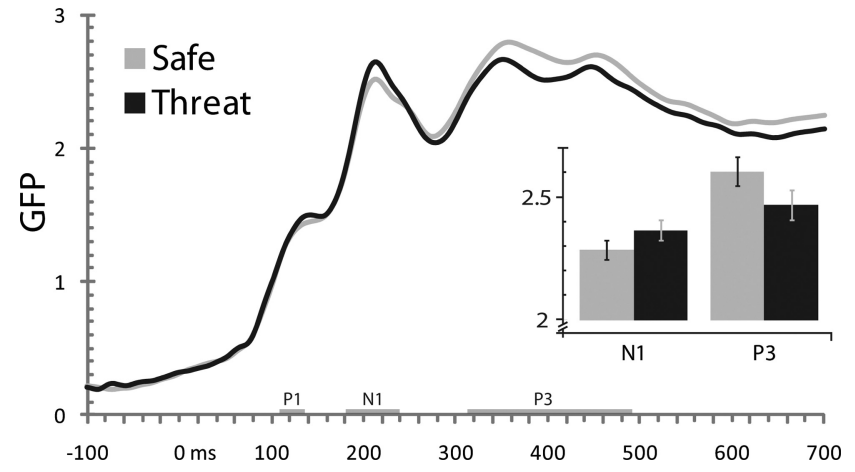

Figure 1. Stress double-dissociated earlier from later task-evoked GFP (SD $\mu$ V). Threat amplified the N1 (184-236 ms) and attenuated the P3 (316-488 ms), without substantially altering the P1 (112-144 ms). Confidence bars indicate the probability of the null hypothesis being rejected by chance; non-overlapping bars indicate $p<0.05$ (Shackman et al., 2010).

global field power (GFP), the SD of voltage across channels. Larger GFP values correspond to stronger underlying local field potentials (i.e., increased neural activity) (Murray et al., 2008). Components were identified by inspection of the grand average ERP and GFP (Fig. 1 and supplemental Fig. S2, available at www.jneurosci.org as supplemental material). Voltages were averaged using symmetric, peak-centered windows: P1 (112-144 ms), N1 (184-236 ms), and P3 (316-488 ms).

Preliminary hypothesis testing was performed using contrasts conducted on GFP (threat vs safe). The hypothesized double dissociation was assessed by testing the Stress (threat, safe) $\times$ Component (early, late) interaction. Components that showed no evidence of stress modulation were omitted from follow-up tests. To identify maximally stress-sensitive regions for each component, electrode-wise $F$ tests comparing mean voltage during threat and safety were conducted. Familywise error (FWE) was controlled at the cluster level using a permutation-based procedure (see the supplemental material, available at www.jneurosci. org) (Nichols and Holmes, 2002). Apparent hemispheric asymmetries ("lateralities") were tested by contrasting mean cluster activity for the target cluster and its homolog, equivalent to the Stress $\times$ Hemisphere interaction (Bonferroni corrected for the number of tests). Permutationbased procedures were also used to identify regions where (1) stress altered mean activity and (2) variation in activity predicted variation in behavior under stress. That is, the latter procedure assessed the jointhypothesis of nonzero mean and individual differences (see the supplemental material, available at www.jneurosci.org). This analysis used Spearman's $\rho$ performed using cluster averages. Corrected $p$ values are reported for regional analyses (see the supplemental material, available at www.jneurosci.org).

\section{Results}

Participants reported experiencing more intense stress under threat $(M=6.5, \mathrm{SD}=20.0)$ than during safety $(M=-19.3$, $\mathrm{SD}=11.6), t_{(31)}=6.7, p<0.001$. They also expressed more intense negative emotion on their faces, indexed by corrugator EMG activity, under threat $(M=-1.0, \mathrm{SD}=0.5)$ than during safety $(M=-1.1, \mathrm{SD}=0.4), t_{(31)}=2.7, p=0.01$ (supplemental Fig. S1, available at www.jneurosci.org as supplemental material). Mean accuracy $(p=0.92)$ and reaction time (RT) $(p=$ 0.09 ) were not reliably affected by threat (see the supplemental material, available at www.jneurosci.org).

Neurally, stress dissociably altered evoked activity during two distinct periods (Fig. 1). Initially, threat increased GFP during the N1 window, $F_{(1,31)}=4.2, p=0.05$. Subsequently, threat decreased GFP during the P3 window, $F_{(1,31)}=5.5, p=0.03$. GFP modulation was not obtained during the P1 window, $F<1$. Underscoring the dissociable impact of stress on successive stages of visual-cognitive processing, a disordinal interaction was ob- 
tained between Stress (threat, safety) and Component (N1, P3), $F_{(1,31)}=15.8, p=$ 0.0004 (Fig. 1).

To determine the most stress-sensitive scalp regions, electrode-wise contrasts were performed for the N1 and P3 (Fig. 2, supplemental Table S1, available at www. jneurosci.org as supplemental material). Permutation-based procedures were used to control FWE across the array. For the $\mathrm{N} 1$, this indicated that threat-induced stress potentiated visually evoked activity in a large cluster of electrodes centered along the zenith of the scalp, $p<0.001$. A second cluster of electrodes in the vicinity of the right mastoid exhibited a similar trend, $p=0.07$. Laterality analyses confirmed that potentiation of visually evoked activity in the right mastoid cluster was stronger than the homologous region of the left-hemisphere, $p=0.001$. For the P3, regional analyses indicated that stress attenuated task-evoked activity across a large cluster of centroparietal electrodes, $p=0.05$.

A final analysis was used to identify regions where mean activity was both sensitive to stress and predictive of individual differences in behavior under stress. This revealed a cluster of right-frontal electrodes that showed both effects, $p=0.02$ (Fig. 3, supplemental Table S2, available at www.jneurosci.org as supplemental material). Here, $\mathrm{P} 3$ activity was attenuated by stress, particularly so in the right hemisphere (laterality test: $p=0.03$ ), and those participants showing greater attenuation exhibited worse performance on the discrimination task, indexed by increased response latencies $(\rho=0.45)$. This effect was not the result of a speed-accuracy trade-off (supplemental Fig. S3, available at www.jneurosci.org as supplemental material). No other clusters emerged for either component for any measure of performance or emotion.

\section{Discussion}

The present findings demonstrate that task-irrelevant stress, elicited by uncertain physical threat, is associated with dissociable effects on earlier sensory processing and later postperceptual processing. This provides new evidence that the sustained stress characteristic of dangerous environments can have fundamentally different consequences for the perception of visual information and the subsequent, task-directed evaluation of that information. These observations are consistent with recent models of stress and anxiety derived from hemodynamic and behavioral studies in humans and molecular studies in monkeys and rodents (Bishop, 2007; Arnsten, 2009).

Threat of shock increased the subjective experience and objective expression of stress, indexed by retrospective ratings and continuously recorded corrugator EMG activity, respectively. Contemporaneously, threat amplified earlier (N1, 184-236 ms)

\section{A N1 Amplification under Stress}

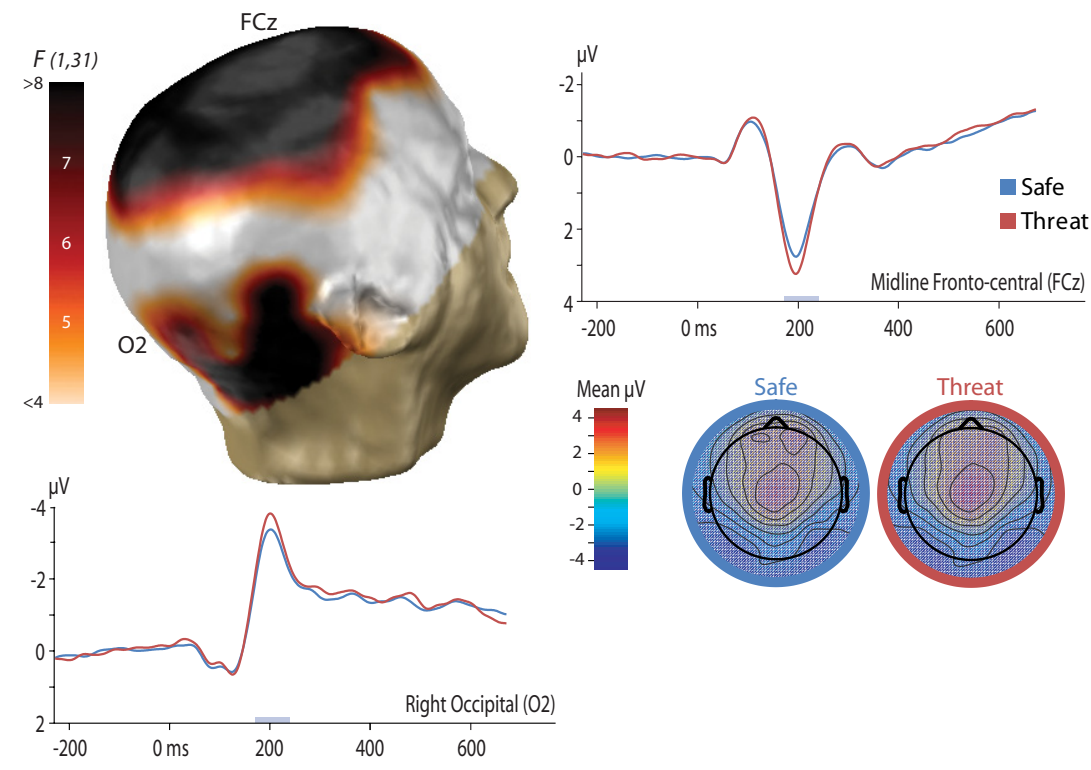

B P3 Attenuation under Stress
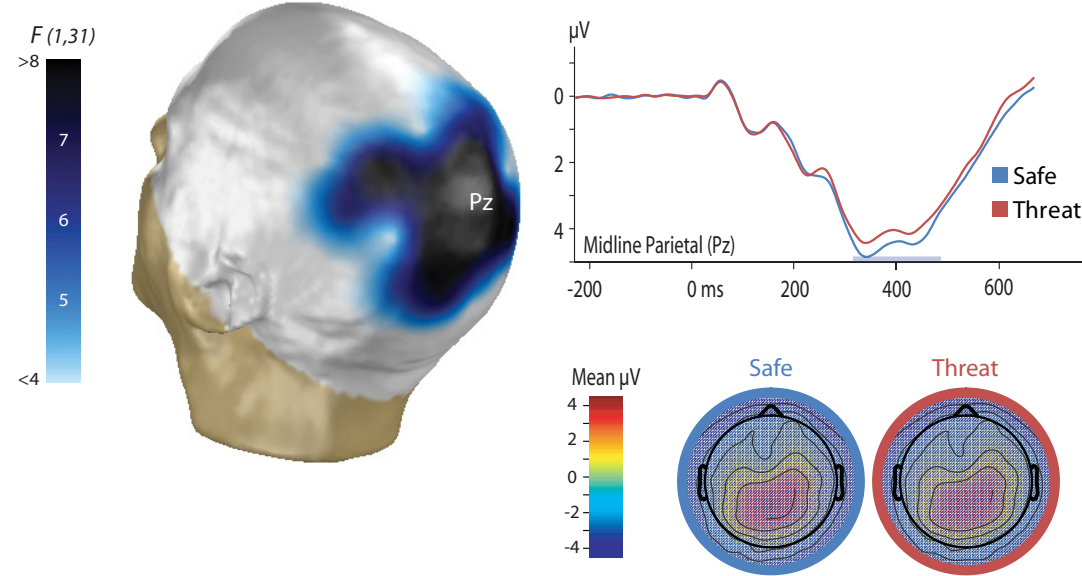

Figure 2. Stress amplified early perceptual processing (N1) and attenuated subsequent postperceptual processing (P3). The N1 and $\mathrm{P} 3$ components are shown in $\boldsymbol{A}$ and $\boldsymbol{B}$, respectively. Three-dimensional topographic maps depict the thresholded threat versus condition. Results are shown for the average reference.

and attenuated later (P3, 316-488 ms) neural activity associated with the processing of emotionally neutral stimuli (Fig. 1). This double dissociation rules out the possibility that these effects are determined by nonspecific changes in arousal or distraction. Nonspecific changes would be expected to exert similar effects on both stages of processing. During both the N1 and P3 windows, threat-induced stress was associated with more pronounced effects at right-hemisphere electrodes (supplemental Table S2, available at www.jneurosci.org as supplemental material). During the P3 window, but not the earlier N1 window, changes in activity predicted stress-induced performance degradation (Fig. 3).

The use of threat of shock to induce stress makes these observations particularly noteworthy, given the potential limitations of alternative manipulations that rely on the intermixed presentation of task cues and threat stimuli (e.g., emotional scenes, faces, or films) in the same perceptual channel. Doing so risks confounding the influence of emotional states or moods, such as stress, with differences in perception (Olofsson et al., 2008) or 


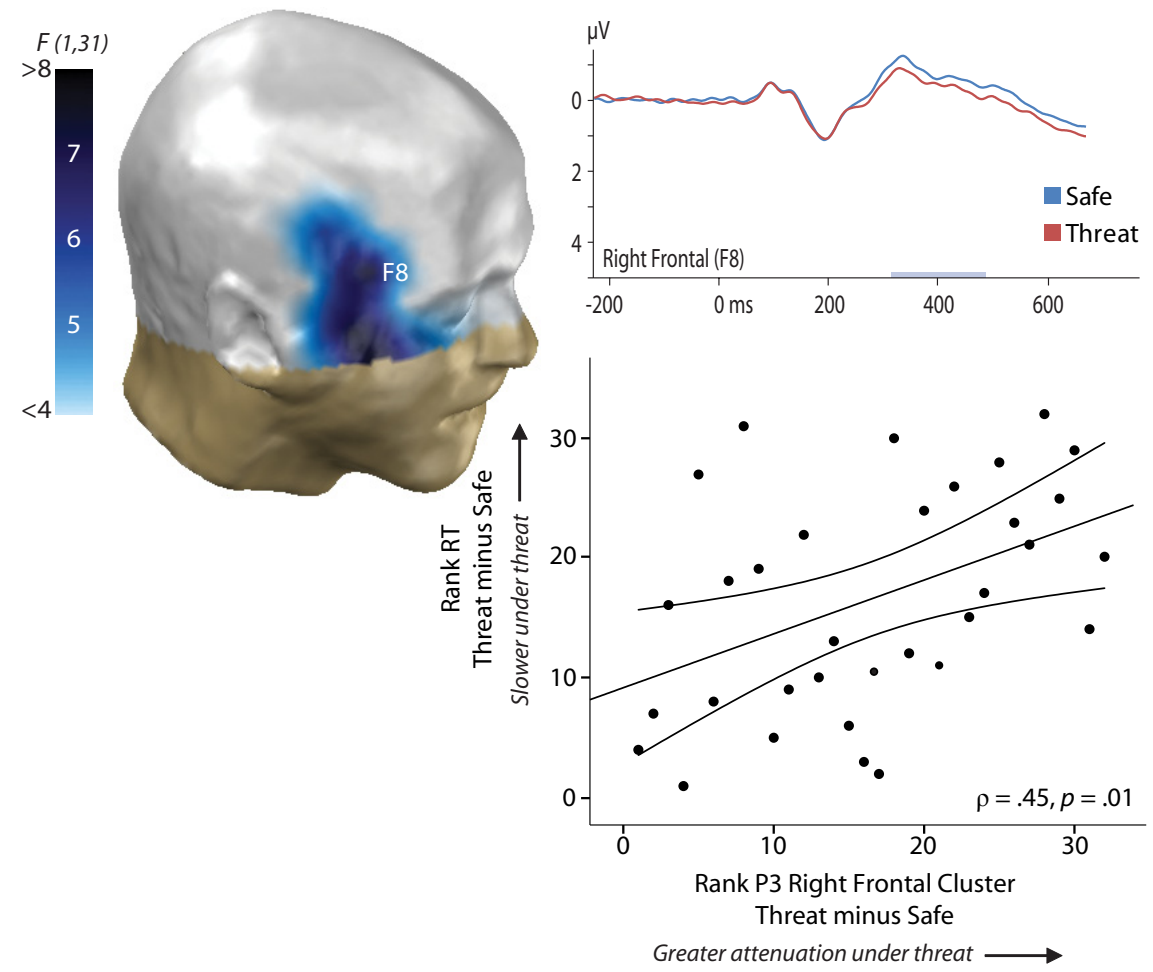

Figure 3. Greater attenuation of the $\mathrm{P} 3$ at right-frontal electrodes predicted reduced task performance. Mean $\mathrm{P} 3$ activity in this cluster was attenuated under threat, depicted in the three-dimensional topographic map. Individuals showing greater P3 attenuation in this cluster exhibited worse performance on the discrimination task, indexed by increased RT, $\rho=0.45$. The joint test was significant, $p=0.02$.

attention (Pereira et al., 2006; Nummenmaa et al., 2009). Compounding this limitation, prior work suggests that briefly presented emotional stimuli do not necessarily produce sustained negative affect (Bradley et al., 1996). This concern is particularly grave in studies where concomitant measures of emotion were not acquired (Shackman et al., 2006).

Stress-induced amplification of the N1 likely stems from modulation of extrastriate cortex-the primary generator of the visually evoked N1 (Mishra and Hillyard, 2009) — by the amygdala or lateral PFC (Deouell and Knight, 2005; Bishop, 2007; Lim et al., 2009; Sabatinelli et al., 2009). Such biasing signals are thought to underlie the increased vigilance and augmented sensory intake characteristically elicited by threatening environments (Davis and Whalen, 2001).

$\mathrm{N} 1$ amplification suggests that stress alters visual attention by increasing the gain on sensory processing. This inference stems in part from the absence of reliable changes in the earlier P1 window (112-144 ms) (Fig. 1). This is potentially informative in light of previously reported dissociations between the P1 and N1 (Mangun and Hillyard, 1991; Mishra and Hillyard, 2009). Although both components have been localized to extrastriate cortex, $\mathrm{P} 1$ is thought to reflect the gating of incoming information via inhibition of unattended or ignored information, whereas $\mathrm{N} 1$ is thought to reflect orienting and discrimination processes that operate via enhancement of incoming information (Hopfinger et al., 2004). These observations suggest that the impact of threatinduced stress on early sensory processing is primarily achieved through increases in sensory gain rather than increased selectivity (cf. Kastner and Ungerleider, 2000). This view is consistent with evidence that the amygdala densely innervates the visual cortices (Freese and Amaral, 2009). Extant anatomical research in nonhuman primates suggests that such connections likely permit ex- citatory feedback modulation (Freese and Amaral, 2009), allowing the amygdala to potentiate visual processing under threat. Interestingly, acute stress seems to increase the sensitivity but decrease the specificity of amygdala reactivity, possibly through a catecholamine-mediated pathway (van Marle et al., 2009). This is consistent with our observation that stress amplified early sensory processing of nonthreatening stimuli. In a genuinely dangerous natural environment, such "false positives" are a small price to pay for the expedited detection of threat.

P3 attenuation likely arises from the disruption of cognitive control processes implemented in frontoparietal regions. The P3 has been conceptualized as an index of selective attention that reflects processes involved in evaluating targets to engage appropriate goal-directed responses (Nieuwenhuis et al., 2005; Duncan et al., 2009). Indeed, P3 amplitude is attenuated in the face of secondary tasks and distractions (Kok, 2001). Other research suggests that it also reflects the downstream consequences of activity in the locus ceruleus-norepinephrine (LCNE) system (Pineda et al., 1989; Nieuwenhuis et al., 2005), the major ascending source of NE to the brain (Berridge, 2008). The LC-NE system is exquisitely sensitive to stress; threat and other stressors amplify NE levels in PFC (Arnsten, 2009), an effect mediated by excitatory projections from the amygdala to LC (Goldstein et al., 1996). These observations suggest that attenuation of the task-evoked P3 reflects (1) the diversion of topdown control to potentially threat-relevant information in the environment, (2) the disruption of top-down control by alterations in catecholaminergic activity, or (3) a combination of these mechanisms.

Our finding that stress attenuated the selective attention processes indexed by the P3 in healthy individuals extends prior work in clinical samples. For instance, the task-related P3 is attenuated in spider phobics in the presence of an uncaged tarantula (Moser et al., 2005). Notably, our results also imply that reduction of the task-evoked $\mathrm{P} 3$ in the face of aversive emotional images (Li et al., 2006; Keil et al., 2007) is at least partially attributable to the negative affect elicited by such images, rather than the reflexive capture of attention.

The impact of threat-induced stress on both earlier perceptual (N1) and later postperceptual (P3) visual processing was reliably exaggerated at electrodes overlying the right hemisphere. This asymmetry is consistent with a large body of work demonstrating that arousing states of stress, such as that elicited by threat of shock (Dalton et al., 2005; Coan et al., 2006) and aversive images (Dolcos and McCarthy, 2006), activate right lateral PFC. The present results are consistent with the hypothesis (Maxwell et al., 2005; Shackman et al., 2009) that this stems from the preferential involvement of the right hemisphere in vigilance/sustained attention (Robertson and Garavan, 2004), reflexive attention (Corbetta et al., 2008), and arousing states of anxiety (Shackman et al., 2006). Another 
possibility is that this reflects neurotransmitter asymmetries (Davidson et al., 1993; Shackman et al., 2006).

The dissociable impact of threat of shock on the N1 and P3 components provides novel support for the hypothesis that stress and anxiety can fundamentally alter the balance of neural processing (Bishop, 2007; Arnsten, 2009). By this account, stress is associated with a catecholamine-mediated shift from a highly controlled task-directed mode, reflecting the influence of prefrontal biasing signals on posterior sensory and motor regions (Miller and Cohen, 2001), to a vigilant threat-assessment mode, reflecting the influence of amygdalar biasing signals (Davis and Whalen, 2001). Consistent with this perspective, we found that stress potentiated the early processing of visual stimuli in extrastriate visual areas, indexed by the N1, but attenuated the subsequent task-directed evaluation of the same stimuli, indexed by the P3.

P3 attenuation was selectively associated with reduced performance of the speeded visual discrimination task under stress, reinforcing the argument that this component more closely reflects postperceptual evaluative processes than the N1. Nevertheless, the fact that stress did not alter mean performance warrants comment. It is likely that this null effect reflects several factors. First, individuals show marked variability in their reactions to stress; often, many participants do not show frank evidence of stress in response to laboratory stressors, such as threat of shock (Shackman et al., 2006). Second, mean performance of the discrimination task was near ceiling, rendering it insensitive to perturbation by stress (Shackman et al., 2006). More generally, this null effect probably reflects the principle that overt behavior is influenced by multiple, convergent neural processes and that alterations in particular stages of the processing stream may or may not be sufficient to shift the behavioral output of this stream. Use of a more potent stressor or sensitive task would be expected to reveal more robust differences in performance.

Because of the practical difficulties of manipulating emotion in the laboratory, little attention has been paid to the impact of stress, fear, and anxiety on the neural circuitry underlying human cognition. This is problematic in light of a growing body of work showing that altered responsiveness to uncertain threat plays a key role in a variety of clinical anxiety disorders (Davis et al., 2010) and maladaptive behaviors (Moberg and Curtin, 2009). The present investigation represents a key step toward addressing this gap. Future research in this area is certain to have substantial benefits for our understanding of how "feeling frazzled" (Arnsten, 1998) influences competition for the control of attention and action.

\section{References}

Arnsten AF (1998) The biology of being frazzled. Science 280:1711-1712.

Arnsten AF (2009) Stress signalling pathways that impair prefrontal cortex structure and function. Nat Rev Neurosci 10:410-422.

Arnsten AF, Goldman-Rakic PS (1998) Noise stress impairs prefrontal cortical cognitive function in monkeys: evidence for a hyperdopaminergic mechanism. Arch Gen Psychiatry 55:362-368.

Berridge CW (2008) Noradrenergic modulation of arousal. Brain Res Rev $58: 1-17$.

Bishop SJ (2007) Neurocognitive mechanisms of anxiety: an integrative account. Trends Cogn Sci 11:307-316.

Bradley MM, Cuthbert BN, Lang PJ (1996) Picture media and emotion: effects of a sustained affective context. Psychophysiology 33:662-670.

Coan JA, Schaefer HS, Davidson RJ (2006) Lending a hand: social regulation of the neural response to threat. Psychol Sci 17:1032-1039.

Corbetta M, Patel G, Shulman GL (2008) The reorienting system of the human brain: from environment to theory of mind. Neuron 58:306-324.
Dalton KM, Kalin NH, Grist TM, Davidson RJ (2005) Neural-cardiac coupling in threat-evoked anxiety. J Cogn Neurosci 17:969-980.

Davidson RJ, Kalin NH, Shelton SE (1993) Lateralized response to diazepam predicts temperamental style in rhesus monkeys. Behav Neurosci 107:1106-1110.

Davis M, Whalen PJ (2001) The amygdala: vigilance and emotion. Mol Psychiatry 6:13-34.

Davis M, Walker DL, Miles L, Grillon C (2010) Phasic vs sustained fear in rats and humans: role of the extended amygdala in fear vs anxiety. Neuropsychopharmacology 35:105-135.

Deouell LY, Knight RT (2005) ERP measures of multiple attention deficits following prefrontal damage. In: Neurobiology of attention (Itty L, Rees G, Tsotos JK, eds), pp 339-344. San Diego: Elsevier.

Dolcos F, McCarthy G (2006) Brain systems mediating cognitive interference by emotional distraction. J Neurosci 26:2072-2079.

Duncan CC, Barry RJ, Connolly JF, Fischer C, Michie PT, Näätänen R, Polich J, Reinvang I, Van Petten C (2009) Event-related potentials in clinical research: guidelines for eliciting, recording, and quantifying mismatch negativity, P300, and N400. Clin Neurophysiol 120:1883-1908.

Erk S, Kleczar A, Walter H (2007) Valence-specific regulation effects in a working memory task with emotional context. Neuroimage 37:623-632.

Fales CL, Barch DM, Burgess GC, Schaefer A, Mennin DS, Gray JR, Braver TS (2008) Anxiety and cognitive efficiency: differential modulation of transient and sustained neural activity during a working memory task. Cogn Affect Behav Neurosci 8:239-253.

Freese JL, Amaral DG (2009) Neuroanatomy of the human amygdala. In: The human amygdala (Whalen PJ, Phelps EA, eds), pp 3-42. New York: Guilford.

Goldstein LE, Rasmusson AM, Bunney BS, Roth RH (1996) Role of the amygdala in the coordination of behavioral, neuroendocrine, and prefrontal cortical monoamine responses to psychological stress in the rat. J Neurosci 16:4787-4798.

Grillon C, Ameli R (1998) Effects of threat of shock, shock electrode placement and darkness on startle. Int J Psychophysiol 28:223-231.

Hopfinger JB, Luck SJ, Hillyard SA (2004) Selective attention: electrophysiological and neuromagnetic studies. In: The cognitive neurosciences, Ed 3 (Gazzaniga MS, ed), pp 561-574. Cambridge, MA: MIT Press.

Kastner S, Ungerleider LG (2000) Mechanisms of visual attention in the human cortex. Annu Rev Neurosci 23:315-341.

Kavaliers M, Choleris E (2001) Antipredator responses and defensive behavior: ecological and ethological approaches for the neurosciences. Neurosci Biobehav Rev 25:577-586.

Keil A, Bradley MM, Junghöfer M, Russmann T, Lowenthal W, Lang PJ (2007) Cross-modal attention capture by affective stimuli: evidence from event-related potentials. Cogn Affect Behav Neurosci 7:18-24.

Kok A (2001) On the utility of P3 amplitude as a measure of processing capacity. Psychophysiology 38:557-577.

Lanteaume L, Khalfa S, Régis J, Marquis P, Chauvel P, Bartolomei F (2007) Emotion induction after direct intracerebral stimulations of human amygdala. Cereb Cortex 17:1307-1313.

Lee H, Shackman AJ, Jackson DC, Davidson RJ (2009) Test-retest reliability of voluntary emotion regulation. Psychophysiology 46:874-879.

Li W, Howard JD, Parrish TB, Gottfried JA (2008) Aversive learning enhances perceptual and cortical discrimination of indiscriminable odor cues. Science 319:1842-1845.

Li X, Li X, Luo Y-J (2006) Differential influences of negative emotion on spatial and verbal working memory: evidence from event-related potential and source current density analysis. Neuroreport 17:1555-1559.

Lim SL, Padmala S, Pessoa L (2009) Segregating the significant from the mundane on a moment-to-moment basis via direct and indirect amygdala contributions. Proc Natl Acad Sci U S A 106:16841-16846.

Mangun GR, Hillyard SA (1991) Modulations of sensory-evoked brain potentials indicate changes in perceptual processing during visual-spatial priming. J Exp Psychol Hum Percept Perform 17:1057-1074.

Maxwell JS, Shackman AJ, Davidson RJ (2005) Unattended facial expressions asymmetrically bias the concurrent processing of nonemotional information. J Cogn Neurosci 17:1386-1395.

Miller EK, Cohen JD (2001) An integrative theory of prefrontal cortex function. Annu Rev Neurosci 24:167-202.

Mishra J, Hillyard SA (2009) Endogenous attention selection during binocular rivalry at early stages of visual processing. Vision Res 49:1073-1080.

Moberg CA, Curtin JJ (2009) Alcohol selectively reduces anxiety but not 
fear: startle response during unpredictable vs. predictable threat. J Abnorm Psychol 118:335-347.

Moser JS, Hajcak G, Simons RF (2005) The effects of fear on performance monitoring and attentional allocation. Psychophysiology 42:261-268.

Murray MM, Brunet D, Michel CM (2008) Topographic ERP analyses: a step-by-step tutorial review. Brain Topogr 20:249-264.

Nichols TE, Holmes AP (2002) Nonparametric permutation tests for functional neuroimaging: a primer with examples. Hum Brain Mapp 15:1-25.

Nieuwenhuis S, Aston-Jones G, Cohen JD (2005) Decision making, the P3, and the locus coeruleus-norepinephrine system. Psychol Bull 131:510-532.

Nummenmaa L, Hyönä J, Calvo MG (2009) Emotional scene content drives the saccade generation system reflexively. J Exp Psychol Hum Percept Perform 35:305-323.

Olofsson JK, Nordin S, Sequeira H, Polich J (2008) Affective picture processing: an integrative review of ERP findings. Biol Psychol 77:247-265.

Pereira MG, Volchan E, Leal de Souza GG, Oliveira L, Campagnoli RR, Pinheiro WM, Pessoa L (2006) Sustained and transient modulation of performance induced by emotional picture viewing. Emotion 6:622-634.

Phelps EA, Ling S, Carrasco M (2006) Emotion facilitates perception and potentiates the perceptual benefits of attention. Psychol Sci 17:292-299.

Pineda JA, Foote SL, Neville HJ (1989) Effects of locus coeruleus lesions on auditory, long-latency, event-related potentials in monkey. J Neurosci 9:81-93.
Qin S, Hermans EJ, van Marle HJ, Luo J, Fernández G (2009) Acute psychological stress reduces working memory-related activity in the dorsolateral prefrontal cortex. Biol Psychiatry 66:25-32.

Robertson IH, Garavan H (2004) Vigilant attention. In: The cognitive neurosciences III, Ed 3 (Gazzaniga MS, ed), pp 563-578. Cambridge, MA MIT Press.

Sabatinelli D, Lang PJ, Bradley MM, Costa VD, Keil A (2009) The timing of emotional discrimination in human amygdala and ventral visual cortex. J Neurosci 29:14864-14868.

Shackman AJ, Sarinopoulos I, Maxwell JS, Pizzagalli DA, Lavric A, Davidson RJ (2006) Anxiety selectively disrupts visuospatial working memory. Emotion 6:40-61.

Shackman AJ, McMenamin BW, Maxwell JS, Greischar LL, Davidson RJ (2009) Right dorsolateral prefrontal cortical activity and behavioral inhibition. Psychol Sci 20:1500-1506.

Shackman AJ, McMenamin BW, Maxwell JS, Greischar LL, Davidson RJ (2010) Identifying robust and sensitive frequency bands for interrogating neural oscillations. Neuroimage 51:1319-1333.

Susskind JM, Lee DH, Cusi A, Feiman R, Grabski W, Anderson AK (2008) Expressing fear enhances sensory acquisition. Nat Neurosci 11:843-850. van Marle HJ, Hermans EJ, Qin S, Fernández G (2009) From specificity to sensitivity: how acute stress affects amygdala processing of biologically salient stimuli. Biol Psychiatry 66:649-655. 